\title{
Optimal Design of a RISE Feedback Controller for a 3-DOF Robot Manipulator Using Particle Swarm Optimization
}

\author{
Marzieh Yazdanzad \\ Dept. of Computer and Electrical Engineering, Babol University of Technology, Babol, Iran \\ Email: s.yazdanzad@stu.nit.ac.ir \\ Alireza Khosravi, Abolfazl Ranjbar N., Pouria Sarhadi \\ Dept. of Computer and Electrical Engineering, Babol University of Technology, Babol, Iran \\ Email: akhosravi@nit.ac.ir, a.ranjbar@nit.ac.ir,pouria.sarhadi@gmail.com
}

\begin{abstract}
This paper presents an application of recently proposed robust integral of the sign of the error (RISE) feedback control scheme for a three degrees-of-freedom (DOF) robot manipulator tracking problem. This method compensates for nonlinear disturbances and uncertainties in the dynamic model, and results in asymptotic trajectory tracking. To avoid selecting parameters of the RISE controller by time-consuming trial and error method, particle swarm optimization (PSO) algorithm is employed. The objective of the PSO algorithm is to find a set of parameters that minimizes the mean of root squared error as the fitness function. The proposed method attains tracking goal, without any chattering in control input. Indeed, the existence of a unique integral sign term in the RISE controller avoids the occurrence of chattering phenomenon that usually happens in sliding mode controllers. Numerical simulations demonstrate the effectiveness of the proposed control scheme.
\end{abstract}

Index Terms - Robust integral of the sign of the error (RISE), Asymptotic tracking, 3degrees-of-freedom robot manipulator, particle swarm optimization (PSO)

\section{INTRODUCTION}

In the past few decades, control of robotic systems has been studied significantly, because of their wide spectrum applications in medicine, aerospace, automotive and other industries. Robot manipulators are of highly nonlinear dynamic systems, which suffer from various uncertainties such as nonlinear friction, payload variation, unknown disturbances, and etc. Several control methods have been presented to attain an accurate tracking control of robot manipulators, such as adaptive control [1,2], sliding mode control [3,4], robust control [5] and neural network techniques $[6,7]$.

Adaptive controllers may cope with the uncertainties in the dynamic model, and present fine tracking performance. However, this technique is restricted to compensate uncertainties which are linear in parameters. Sliding mode control is one of the effective strategies to control uncertain nonlinear systems. Main advantage of this method is strong robustness against system uncertainties, parameter variations and nonlinear disturbances [8]. But this technique, has some drawbacks including creating chattering phenomenon due to the discontinuous nature of control law, and the requirement of knowing an exact knowledge of the system dynamics [9].

It is difficult to obtain an exact mathematical model of robotic systems, due to modeling uncertainties, parameters variations, and etc. In recent years, intelligent control approaches such as neural network (NN)-based control and fuzzy control in robotic systems control, have received considerable attention [10,11,12]. Neural networks are almost able to approximate nonlinear continuous functions. They are therefore powerful tools to compensate for uncertainties, without knowing a complete knowledge of the plant [13]. Lewis et al. proposed a multilayer neural network controller for a robot manipulator which guarantees trajectory tracking performance [14]. However due to the NN functional reconstruction error, this type of controllers only achieve uniformly ultimately bounded (UUB) stability results. In order to eliminate the approximation error of NN-based controllers and achieving asymptotic tracking, Wai presented a sliding-mode neural network controller for rigid link manipulators [15]. In spite of obtaining asymptotic results, existence of a sign function in the robust term results in the destructive chattering phenomenon.

Recently, a new feedback control strategy called robust integral of the sign of the error (RISE) is proposed in [16] which compensates for disturbances or uncertainties of dynamic system, by generating a continuous control signal [17,18]. In [19], Patre et al. utilized this method to develop a tracking controller in presence of additive disturbances and parametric uncertainties. The RISE method is a high gain feedback tool. Motivated by this issue, $_{2}$ in $[20,21]$, a NN-based feed-forward term is combined with the RISE feedback term in order to reduce the gain values of RISE feedback and to yield asymptotic tracking results. Also Shicheng Wang et al. in [22], designed a RISE based NN controller for a spacecraft formation within the leader follower architecture. 
Particle swarm optimization (PSO) is a relatively new evolutionary computation technique inspired by the social behavior of birds flocking and fish schooling. It was first introduced by Kennedy and Eberhart in 1995 [23]. In fact, PSO is a population-based search method that can be used to find the optimal solution in a multidimensional search space. Optimization of the gains of a state feedback controller for a flexible manipulator, by PSO algorithm is presented in [24]. Also in [25], using PSO algorithm, an optimal design for a 6-DOF parallel manipulator has presented. Unlike other optimization methods, PSO gains from advantages such as simplicity of implementation and fast convergence.

In this paper, using the new RISE feedback control strategy, an asymptotic tracking is obtained for an uncertain nonlinear 3-DOF rigid link robot system. PSO algorithm is applied to tune gains of the RISE controller by minimizing the root mean squared error of the robot. The obtained results represent satisfactory performance of the controller.

The remainder of this paper is organized as follows. First, in Section 2, nonlinear model of a 3-degree of freedom robot manipulator is presented. Then, the RISE feedback control scheme is introduced in section 3. In section 4, PSO optimization technique is described. Simulation results for the 3-DOF robot manipulator are provided in section 5, and eventually, conclusions are given in section 6 .

\section{DyNAMICS OF THREE LINK RoBOT}

The dynamics of a general rigid link manipulator having $n$-degree of freedom in free space is as follows:

$$
M(q) \ddot{q}+C(q, \dot{q}) \dot{q}+G(q)+\tau_{d}=\tau
$$

This dynamic equation is obtained by means of the Lagrangian approach. In (1) for the rigid 3-DOF robot manipulator $q(t), \dot{q}(t)$ and $\ddot{q}(t) \in R^{3}$ are the position, velocity and acceleration of the joints, respectively. $M(q) \in R^{3 \times 3}$ is the positive definite inertia matrix whilst $C(q, \dot{q}) \in R^{3 \times 3}$ represents the Coriolis/centripetal matrix, and $G(q) \in R^{3}$ expresses the gravity vector. $\tau_{d} \in$ $R^{3}$ denotes the vector of disturbances and un-modeled dynamics, and $\tau \in R^{3}$ denotes the input torque vector applied to the joints. Fig. 1 shows the 3-link robot manipulator.

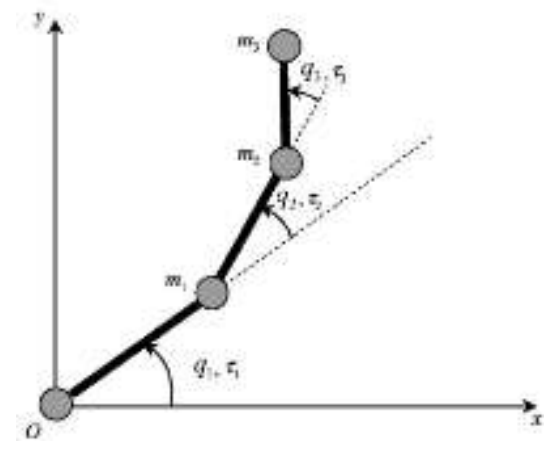

Fig. 1. Three link planar robot
In this system the inertia matrix $M(q)$, the Coriolis/centripetal matrix $C(q, \dot{q})$ and the gravity vector $G(q)$ are as follows [26]:

$$
\begin{aligned}
& M(q, \dot{q})=\left[\begin{array}{lll}
M_{11} & M_{12} & M_{13} \\
M_{21} & M_{22} & M_{23} \\
M_{31} & M_{32} & M_{33}
\end{array}\right], \\
& \text { with } \\
& M_{11}=2\left(d_{1}+d_{2}+d_{3}\right)+2 d_{4} c_{2}+2 d_{5} c_{23}+ \\
& 2 d_{6} c_{3}, \\
& M_{12}=2\left(d_{2}+d_{3}\right)+d_{4} c_{2}+d_{5} c_{23}+2 d_{6} c_{3}, \\
& M_{13}=2 d_{3}+d_{5} c_{23}+d_{6} c_{3}, \\
& M_{21}=M_{12}, \\
& M_{22}=2\left(d_{2}+d_{3}\right)+2 d_{6} c_{3}, \\
& M_{23}=2 d_{3}+d_{6} c_{3}, \\
& M_{31}=M_{13}, M_{32}=M_{23}, M_{33}=2 d_{3},
\end{aligned}
$$

and

$$
C(q, \dot{q}) \dot{q}=\left[\begin{array}{lll}
C_{11} & C_{12} & C_{13} \\
C_{21} & C_{22} & C_{23} \\
C_{31} & C_{32} & C_{33}
\end{array}\right]\left[\begin{array}{l}
\dot{q}_{1} \\
\dot{q}_{2} \\
\dot{q}_{3}
\end{array}\right],
$$

where $C_{i j},(i=1,2,3, j=1,2,3)$ are defined as follows:

$$
\begin{aligned}
& C_{11}=-\dot{q}_{2} d_{4} s_{2}-d_{5} s_{23}\left(\dot{q}_{2}+\dot{q}_{3}\right)-d_{6} s_{3} \dot{q}_{3}, \\
& C_{12}=-d_{4} s_{2}\left(\dot{q}_{1}+\dot{q}_{2}\right)-d_{5} s_{23}\left(\dot{q}_{1}+\dot{q}_{2}+\dot{q}_{3}\right) \\
& -d_{6} s_{3} \dot{q}_{3} \text {, } \\
& C_{13}=-\left(d_{5} s_{23}+d_{6} s_{3}\right)\left(\dot{q}_{1}+\dot{q}_{2}+\dot{q}_{3}\right) \text {, } \\
& C_{21}=\left(d_{4} s_{2}+d_{5} s_{23}\right) \dot{q}_{1}-d_{6} s_{3} \dot{q}_{3} \text {, } \\
& C_{22}=-d_{6} s_{3} \dot{q}_{3} \text {, } \\
& C_{23}=-d_{6} s_{3}\left(\dot{q}_{1}+\dot{q}_{2}+\dot{q}_{3}\right) \text {, } \\
& C_{31}=d_{5} s_{23} \dot{q}_{1}+d_{6} s_{3}\left(\dot{q}_{1}+\dot{q}_{2}\right) \text {, } \\
& C_{32}=d_{6} s_{3}\left(\dot{q}_{1}+\dot{q}_{2}\right), C_{33}=0 \text {, } \\
& \text { and } \\
& G(q)=\left[\begin{array}{l}
g_{1} \\
g_{2} \\
g_{3}
\end{array}\right], \\
& g_{1}=\frac{1}{2} a_{1} c_{1} m_{1} g+\left(a_{1} c_{1}+\frac{1}{2} a_{2} c_{12}\right) m_{2} g+\left(a_{1} c_{1}\right. \\
& \left.+a_{2} c_{12}+\frac{1}{2} a_{3} c_{123}\right) m_{3} g \text {, } \\
& g_{2}=\left(\frac{1}{2} a_{2} c_{12}\right) m_{2} g+\left(a_{2} c_{12}+\frac{1}{2} a_{3} c_{123}\right) m_{3} g \text {, } \\
& g_{3}=\left(\frac{1}{2} a_{3} c_{123}\right) m_{3} g \text {. }
\end{aligned}
$$

where $q=\left[\begin{array}{lll}q_{1} & q_{2} & q_{3}\end{array}\right]^{T}$ and $\dot{q}=\left[\begin{array}{lll}\dot{q}_{1} & \dot{q}_{2} & \dot{q}_{3}\end{array}\right]^{T}$ contain the joints displacement and velocities, respectively. $m_{i}$ and $a_{i}$ are the masses and lengths of the joints. Meanwhile $s_{i}, c_{i}, s_{i j}, c_{i j}$ and $c_{i j k}(i=1,2,3, j=1,2,3, k=1,2,3)$ denote $\sin \left(q_{i}\right), \cos \left(q_{i}\right), \sin \left(q_{i}+q_{j}\right), \cos \left(q_{i}+\right.$ $\left.q_{j}\right), \cos \left(q_{i}+q_{j}+q_{k}\right)$, respectively, whilst parameters $d_{i}, i=1, \ldots, 6$ are defined as follows: 


$$
\begin{aligned}
& d_{1}=\frac{1}{2}\left[\left(\frac{1}{4} m_{1}+m_{2}+m_{3}\right) a_{1}^{2}+I_{o 1}\right] \\
& d_{2}=\frac{1}{2}\left[\left(\frac{1}{4} m_{2}+m_{3}\right) a_{2}^{2}+I_{o 2}\right] \\
& d_{3}=\frac{1}{2}\left[\left(\frac{1}{4} m_{3}\right) a_{3}^{2}+I_{o 3}\right] \\
& d_{4}=\left(\frac{1}{2} m_{2}+m_{3}\right) a_{1} a_{2} \\
& d_{5}=\frac{1}{2} m_{3} a_{1} a_{3} \\
& d_{6}=\frac{1}{2} m_{3} a_{2} a_{3} .
\end{aligned}
$$

where $I_{o i}, i=1,2,3$ denotes the moment of inertia of $i^{\text {th }}$ joint [26].

It is assumed that $q(t)$ and $\dot{q}(t)$ are measurable and $M(q), C(q, \dot{q}), G(q)$ and $\tau_{d}(t)$ are unknown. Furthermore the following properties for the dynamic model of the robot are hold:

Property 1: The inertia matrix $M(q)$ is symmetric, positive definite and holds true the following inequality:

$$
m_{1}\|y\|^{2} \leq y^{T} M(q) y \leq \bar{m}(q)\|y\|^{2} \forall y \in R^{3}
$$

where $m_{1} \in R$ is a known positive constant, $\bar{m}(q) \in R$ is a known positive function and $\|$.$\| represents the$ standard Euclidean norm.

Property 2: If $q(t), \dot{q}(t) \in \mathcal{L}_{\infty}$, then $C(q, \dot{q}), G(q)$ are bounded. Further, if $q(t), \dot{q}(t) \in \mathcal{L}_{\infty}$, then the first and second partial derivatives of the elements of $M(q), C(q, \dot{q}), G(q)$ with respect to $q(t)$ exist and are bounded, and the first and second partial derivatives of the elements of $C(q, \dot{q})$ with respect to $\dot{q}(t)$ exist and are bounded as well.

Property 3: The nonlinear disturbance term and its first two time derivatives are bounded i.e., $\tau_{d}(t), \dot{\tau}_{d}(t), \ddot{\tau}_{d}(t) \in \mathcal{L}_{\infty}$.

\section{RISE FEEDBACK CONTROLLER}

Recently a novel feedback control scheme called robust integral of the sign of the error (RISE) is proposed in [16]. This method compensates for the disturbances or uncertainties of dynamic system using a continuous control signal. Using this technique, an asymptotic tracking is achieved without chattering problem that usually occurs in sliding mode controllers.

It is assumed that the desired trajectory $q_{d}(t) \in R^{n}$, is designed such that $q_{d}{ }^{(i)}(t) \in \mathcal{L}_{\infty}, i=1,2, \ldots, 5$. In order to design a controller for the robot manipulator, with the aim of guaranteeing tracking of a desired time-varying trajectory, $q_{d}(t) \in R^{n}$, by the system, in spite of uncertainties and bounded disturbances in the dynamic model, a position tracking error, denoted by $e_{1}(t) \in R^{n}$, is defined as: $e_{1}=q_{d}-q$

The filtered tracking errors denoted by $e_{2}(t)$ and $r(t) \in R^{n}$ are also defined as:

$$
\begin{aligned}
& e_{2}=\dot{e}_{1}+\alpha_{1} e_{1} \\
& r=\dot{e}_{2}+\alpha_{2} e_{2}
\end{aligned}
$$

where $\alpha_{1} \in R^{n \times n}$ is a positive constant matrix and $\alpha_{2} \in R$ is a positive constant.

The continuous RISE feedback control law to attain the mentioned control objective is as follows [16]:

$$
\begin{aligned}
\tau(t)=\left(k_{s}+1\right) e_{2} & (t)-\left(k_{s}+1\right) e_{2}(0) \\
& +\int_{0}^{t}\left[\left(k_{s}+1\right) \alpha_{2} e_{2}(\sigma)\right. \\
& \left.+\beta_{1} \operatorname{sgn}\left(e_{2}(\sigma)\right)\right] d \sigma
\end{aligned}
$$

Where $k_{s}$ and $\beta_{1} \in R$ are positive constant control gains and $\operatorname{sgn}($.$) is the standard sign function.$

\section{Particle SWARM Optimization TeChNiQue}

\section{A. PSO Algorithm}

PSO is a population-based optimization method inspired by the observation of social behavior of bird flocking. This method consists of a swarm of particles, where each particle represents a potential solution. In a PSO system, each particle flies through the multidimensional search space to adjust its position according to its own flying experience as well as the flying experience of its neighboring particle. In other word, each particle is considered as a point in search space, in which we try to find an optimal location.

Each particle $\mathrm{i}$ has a position vector $X_{i}=$ $\left(x_{i 1}, x_{i 2}, \ldots, x_{i D}\right)$, and a velocity vector $V_{i}=$ $\left(v_{i 1}, v_{i 2}, \ldots, v_{i D}\right)$, where $\mathrm{D}$ represents dimension of search space. Similar to the other optimization techniques, PSO requires a fitness function to evaluate performance of each particle. Further, each particle contains a memory to store the best position in the search space ever seen by it. Velocity and position are initialized with random vectors of the corresponding dimension. Trajectory of each particle in the search space is adjusted by updating the velocity, according to the best position attained so far for it, denoted by $p$ Best, and also the best position gained by any particle in the swarm, denoted by gBest, so far. The updating rule for the velocity and position of the $i^{\text {th }}$ particle on dimension $\mathrm{d}$, is as follows:

$$
\begin{aligned}
& V_{i d}=w v_{i d}+c_{1} r_{1 d}\left(p B e s t_{i d}-x_{i d}\right) \\
& +c_{2} r_{2 d}\left(g \text { Best }_{d}-x_{i d}\right) \\
& V_{i d}=\left\{\begin{array}{rc}
V_{d}^{\max }, & v_{i d}>V_{d}^{\max } \\
-V_{d}^{\max }, & v_{i d}<-V_{d}^{\max }
\end{array}\right. \\
& x_{i d}=x_{i d}+v_{i d}
\end{aligned}
$$

where $w$ is inertia weight that suitable selection of it provides a balance between local and global search ability. $c_{1}$ And $c_{2}$ are positive constants that represent the 
cognitive and social acceleration factors, respectively. $r_{1 d}$ and $r_{2 d}$ are two uniformly distributed random numbers in the range $[0,1]$. pBest $t_{i d}$ denotes the position with the best fitness value, detected so far for the $i^{\text {th }}$ particle. gBest Represents the best position obtained by the population. Furthermore $V_{i \max }=\left(v_{i 1}^{\max }, v_{i 2}^{\max }, \ldots, v_{i D}^{\max }\right)$ represents an upper bound on the absolute amount of the velocity of the $i^{\text {th }}$ particle. Fig. 2 indicates the flowchart of the PSO algorithm.

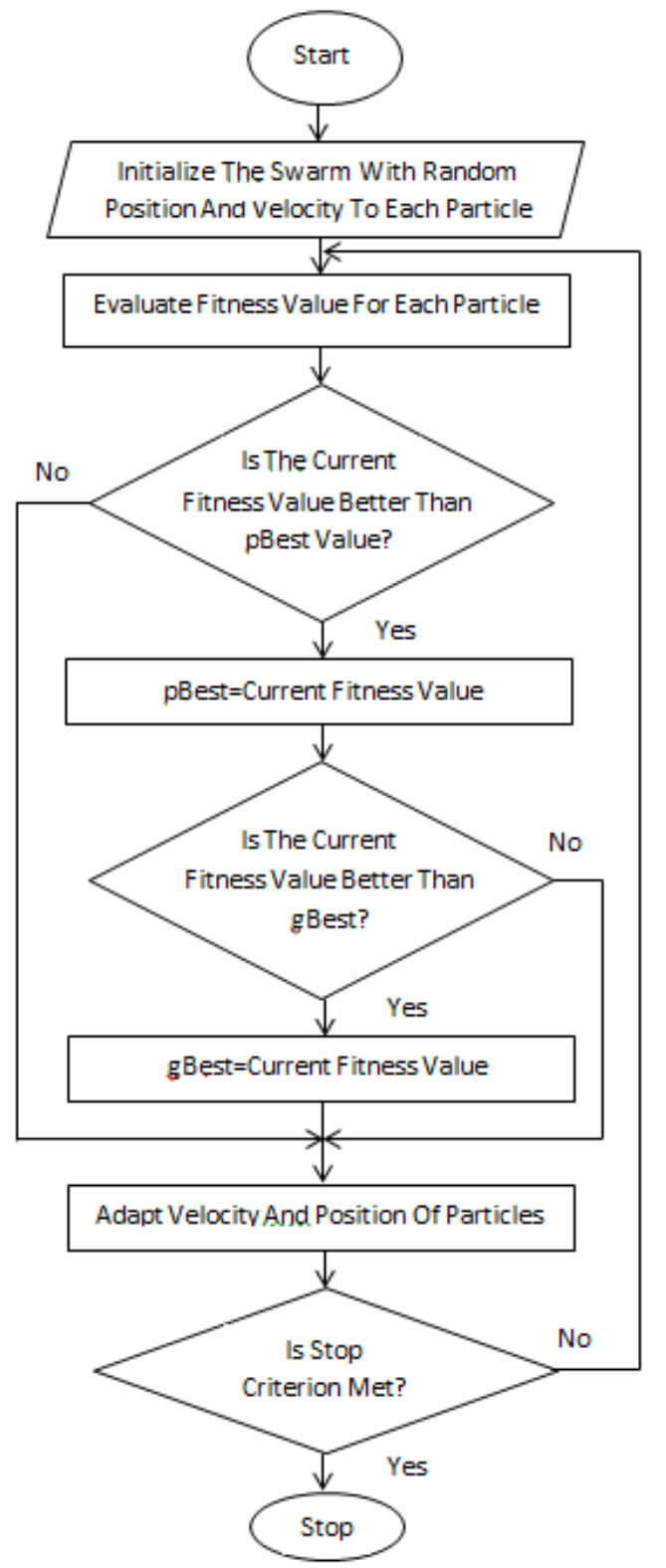

Fig. 2. Flowcharts of particle swarm optimization algorithm [23]

\section{B. Fitness Function}

In this section PSO algorithm is applied to the RISE feedback controller for a three rigid link robot manipulator. The objective is to obtain the values of RISE controller gains $k_{s}, \beta_{1}$ and $\alpha_{2}$ by minimization of the objective function. In this paper, the mean of root of squared error is considered as the cost function, which for $i^{\text {th }}$ particle is as follows:

$$
=\frac{1}{N} \sum_{i=1}^{N} \sqrt{e_{l 1}^{2}(i)+e_{l 2}^{2}(i)+e_{l 3}^{2}(i)}
$$

where $e_{l 1}(i), e_{l 2}(i), e_{l 3}(i)$, are the trajectory tracking error of $i^{\text {th }}$ sample for the first, second and third joint of the robot. $N$ is the number of samples and $k$ is the iteration number.

\section{Simulation RESUlts}

In this section, a numerical simulation for tracking control of a 3-DOF rigid three link manipulator is performed to evaluate the validity of the proposed controller. The numerical values of the robot manipulator parameters are listed in Table 1.

Table 1. Simulation Parameters of Robot

\begin{tabular}{|cccc|}
\hline & $\begin{array}{c}\text { Mass } \\
\left(\boldsymbol{m}_{\boldsymbol{i}}, \mathbf{K g}\right)\end{array}$ & $\begin{array}{c}\text { Link } \\
\left(\boldsymbol{a}_{\boldsymbol{i}}, \boldsymbol{m}\right)\end{array}$ & $\begin{array}{c}\text { Moment of inertia } \\
\left(\boldsymbol{I}_{\boldsymbol{o i}}, \boldsymbol{K g ~ m}^{2}\right)\end{array}$ \\
Joint 1 & 1.2 & 0.5 & $43.33 \times 10^{-3}$ \\
Joint 2 & 1.5 & 0.4 & $25.08 \times 10^{-3}$ \\
Joint 3 & 3.0 & 0.3 & $32.67 \times 10^{-3}$ \\
\hline
\end{tabular}

The desired trajectory to be tracked by links is considered as $q_{d}=[\sin (t) \cos (t) \sin (t+(\pi / 3))]^{T}$. Furthermore external disturbances of $\tau_{d 1}=$ $0.2 \sin (2 t), \tau_{d 2}=0.1 \cos (2 t), \tau_{d 3}=0.1 \sin (t) \quad$ are applied to the system. Initial position and velocity of the joints are set to zero. According to [27], $\chi$ which is called the constriction factor, is defined as follows:

$$
\begin{aligned}
\chi=\frac{2}{\varphi-2+\sqrt{\varphi^{2}-4 \varphi}}, \varphi \\
=\varphi_{1}+\varphi_{2}, \varphi>4
\end{aligned}
$$

In the PSO algorithm parameters $\chi, \varphi_{1}$ and $\varphi_{2}$ are set to $0.7298,2.05$ and 2.05 , respectively. The parameters $w, c_{1}$ and $c_{2}$ are defined according to the following equations:

$$
w=\chi, c_{1}=\chi \varphi_{1}, c_{2}=\chi \varphi_{2}
$$

Gains of the RISE controller are continuously adjusted via PSO algorithm, to minimize the given objective

\begin{tabular}{|c|c|}
\hline Parameter & Value \\
\hline Population Size & 20 \\
\hline No. of Iterations (k) & 50 \\
\hline$v_{\max }$ & 3 \\
\hline$w$ & 0.7298 \\
\hline$c_{1}$ & 1.4962 \\
\hline$c_{2}$ & 1.4962 \\
\hline Lower $\operatorname{Bound}\left(k_{s}, \beta_{1}, \alpha_{2}\right)$ & $\left(\begin{array}{lll}0 & 0 & 0\end{array}\right)$ \\
\hline Upper Bound $\left(k_{s}, \beta_{1}, \alpha_{2}\right)$ & $\left(\begin{array}{lll}30 & 30 & 30\end{array}\right)$ \\
\hline
\end{tabular}
function. Values of tuning parameters of the PSO algorithm are shown in Table 2.

Table 2. PSO Tuning Parameters 
The values of the RISE feedback controller gains obtained using PSO algorithm are given in Table 3.

Table 3. Results of Gains Obtained

\begin{tabular}{|cccc|}
\hline $\boldsymbol{k}_{\boldsymbol{s}}$ & $\boldsymbol{\beta}_{\mathbf{1}}$ & $\boldsymbol{\alpha}_{\mathbf{2}}$ & Best Cost \\
25.32 & 16.7 & 29.3 & 0.001139 \\
\hline
\end{tabular}

Simulation results are shown in Figs. 3 to 5.

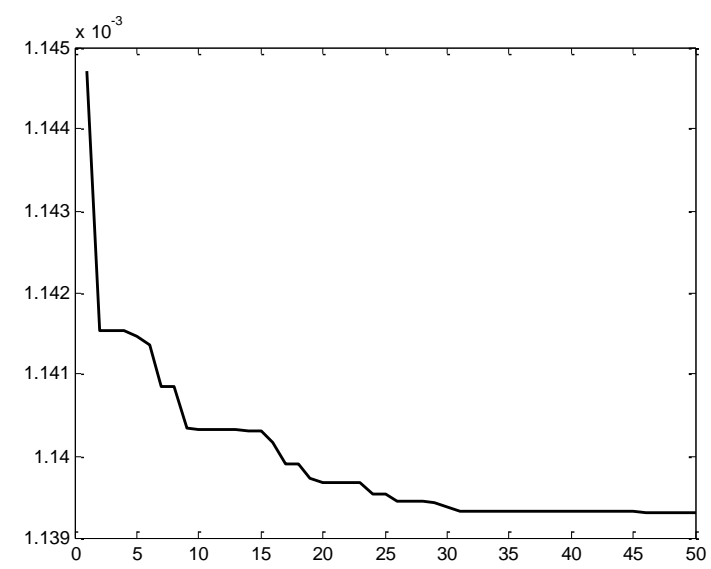

Fig. 3. Fitness function values for 50 iterations

Fig. 3 shows the fitness function trend, which is reduced to find the gain values of the RISE controller.

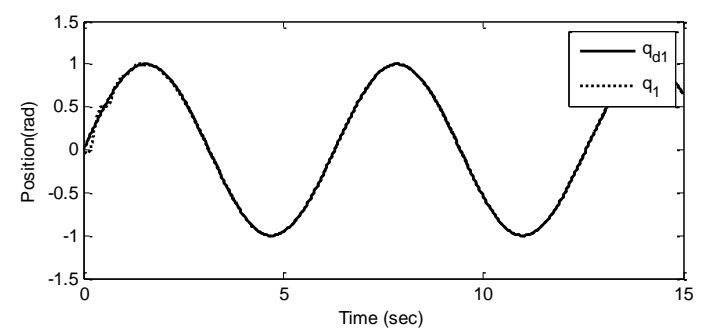

4. a. Trajectory tracking of joint angle 1

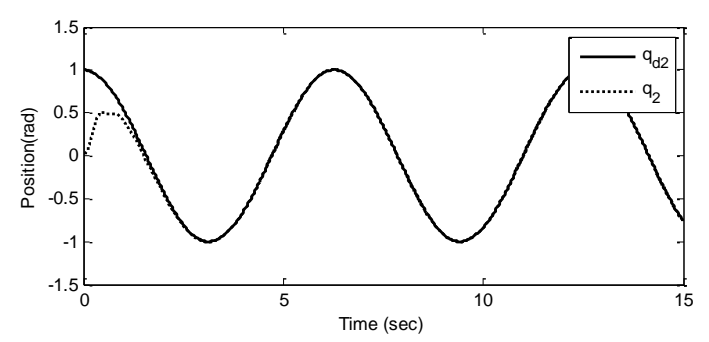

4. b. Trajectory tracking of joint angle 2

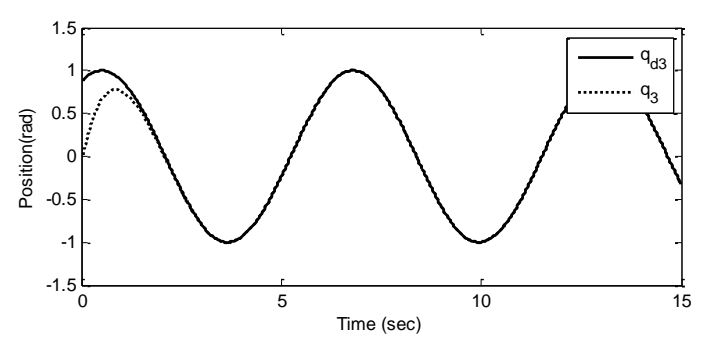

4. c. Trajectory tracking of joint angle 3

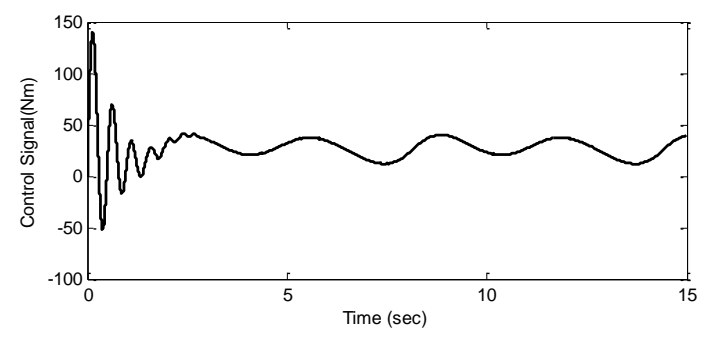

4. d. Control effort for joint 1

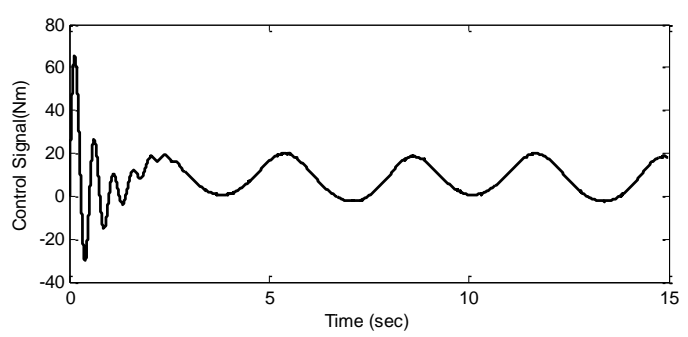

4. e. Control input for joint 2

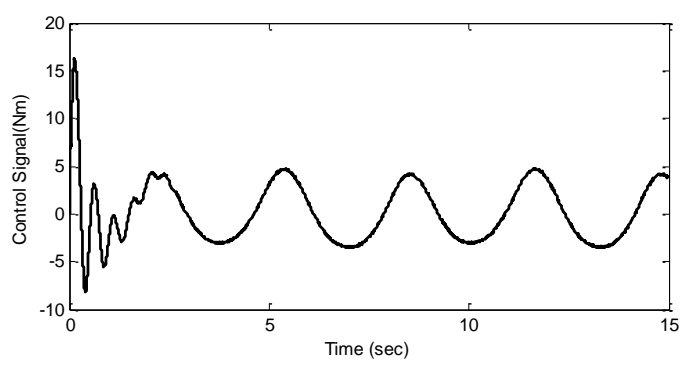

4. f. Control input for joint 3

Fig. 4. Tracking position and control input of joints 1, 2 and 3

The trajectory tracking simulation results of all three joints of the robot and their applied corresponding control inputs are shown in Fig. 4. As it can be seen from the figures, the control objective is successfully achieved. Furthermore, the proposed controller generates a continuous control effort, which prevents chattering phenomenon.

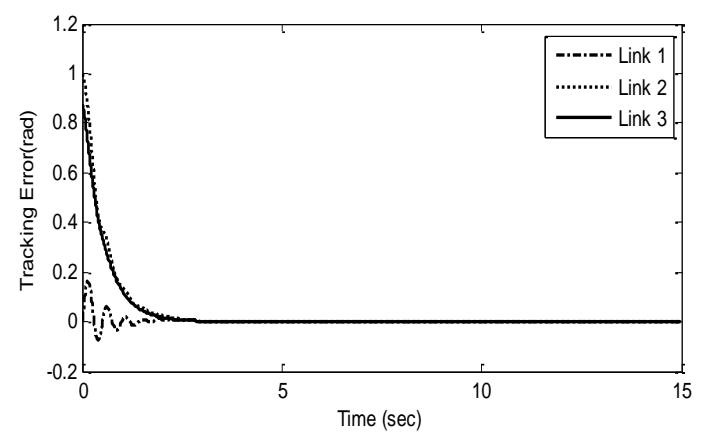

Fig. 5. Tracking error of joints 1,2 and 3

Fig. 5 indicates the position tracking error for all three links of the robot, which confirms that asymptotic tracking is achieved even with the external disturbances. 


\section{CONCLUSION}

In this paper, a continuous control scheme called RISE feedback is utilized for tracking problem of a 3-DOF rigid three link manipulator. An intelligent tuning of the RISE controller parameters is conducted by using particle swarm optimization algorithm. The controller parameters are adjusted while PSO minimizes the mean of root of squared error. The proposed controller compensates for uncertainties and bounded external disturbances without any chattering in the control input. Simulation results have demonstrated significance of the proposed controller together with the capability to provide an asymptotic tracking performance.

\section{REFERENCES}

[1] R. Colbaugh, K. Glass, "Adaptive tracking control of rigid manipulators using only position measurements," Journal of Robot and System vol. 14 no. 1, pp. 9-26, 1997.

[2] M. Erlic andW. Lu, "A reduced-order adaptive velocity observer for manipulator control," IEEE Trans. Robot. Automat., vol. 11, pp. 293-303, Mar. 1995.

[3] F. Piltan, A. Nabaee, M. Ebrahimi, M. Bazregar, "Design Robust Fuzzy Sliding Mode Control Technique for Robot Manipulator Systems with Modeing Uncertainties" I.J. Information Technology and Computer Science, vol. 8, pp. 123-135, 2013.

[4] Y.-W. Liang, S.-D. Xu, D.-C. Liaw, and C.-C. Chen, "A study of T-S model-based SMC scheme with application to robot control," IEEE Trans. Ind. Electron., vol. 55, no. 11, pp. 3964-3971, Nov. 2008.

[5] Guangjun Liu ,Andrew A, Goldenberg, "Robust Control of Robot Manipulators Based on Dynamics Decomposition" IEEE Trans. Robotics and automation, vol.13, pp. 783-789, October, 1997

[6] R.A. Teixeira, A.D. Braga, and B.R. DeMenezes, "Control of a Robotic Manipulator Using Artificial Neural Networks with On-line Adaptation," Neural Processing Letters, vol. 12, pp. 19-31, 2000.

[7] L.B. Gutierrez, F.L. Lewis, J.A. Lowe, "Implementation of a neural network tracking controller for a single Texible link: comparison with PD and PID controller," IEEE Trans. Ind. Electron.vol. 45 307, 1998.

[8] X. Yu, O. Kaynak, "Sliding-Mode Control with Soft Computing: A Survey," IEEE Trans. On Industrial Electronics, vol. 56, no. 9, September, 2009.

[9] .I. Boiko, et al., "Analysis of chattering in systems with second-order sliding modes," IEEE Transactions on Automatic Control, vol. 52, pp. 2085-2102, 2007.

[10] R. J. Wai and M. C. Lee, "Intelligent optimal control of single-link flexible robot arm," Industrial Electronics, IEEE Transactions on, vol. 51, pp. 201-220, 2004.

[11] M. Mirzadeh, M. Haghighi, S. Khezri, J. Mahmoodi, H. Karbasi, "Design Adaptive Fuzzy Inference Controller for 2-DOF Robot Manipulator" I.J. Information Technology and Computer Science, vol. 6, pp. 31-38, May,.2014.

[12] Rong-jong Wa and Po-Chen Chen, "Robust Neural-FuzzyNetwork Control for Robot Manipulator Including Actuator Dynamics," IEEE Trans. Indst. Elect. vol. 53, no. 4, Aug. 2006.

[13] H.D. Patino, R. Carelli, B.R. Kuchen, "Neural networks for advanced control of robot manipulators," IEEE Transactions on Neural Networks vol. 13 no. 2 pp. 343$354,2002$.
[14] F.L. Lewis, A. Yesildirek, K. Liu, "Multilayer neural-net robot controller with guaranteed tracking performance," IEEE Trans. Neural Networks vol. 7, 388, 1996.

[15] R.J. Wai, "Tracking control based on neural network strategy for robot manipulator," Neuro computing vol. 51, pp. 425-445, 2003.

[16] B. Xian, D.M. Dawson, M.S. de Queiroz, J. Chen, "A continuous asymptotic tracking control strategy for uncertain multi-input nonlinear systems," IEEE Transactions on Automatic Control vol. 49, no. 7, pp. 1206-1211, 2004.

[17] C. Makkar, G. Hu, W. G. Sawyer, and W. E. Dixon, "Lyapunov-based tracking control in the presence of uncertain nonlinear parameterizable friction," IEEE Trans. Automat. Control, vol. 52, no. 10, Oct. 2007.

[18] Z. Cai, M. S. de Queiroz, and D. M. Dawson, "Robust adaptive asymptotic tracking of nonlinear systems with additive disturbance," IEEE Trans. Automat. Control, vol. 51, no. 3, Mar. 2006.

[19] P.M. Patre, W. MacKunis, W.E. Dixon, "Asymptotic tracking for uncertain dynamic systems via a multilayer NN feed-forward and RISE feedback control structure," IEEE Transactions on Automatic Control, vol. 53, no. 9, pp. 2180-2185, 2008.

[20] W. MacKunis, P. M. Patre, M. K. Kaiser, and W. E. Dixon, "Asymptotic tracking for aircraft via robust and adaptive dynamic inversion methods," IEEE Trans. Cont. Syst. Technol., 2010.

[21] P. M. Patre, W. MacKunis, C. Makkar, and W. E. Dixon, "Asymptotic tracking for systems with structured and unstructured uncertainties," IEEE Trans. Cont. Syst. Technol., vol. 16, no. 2, Mar. 2008.

[22] S. Wang, H. Min, F. Sun, J. Zhang, "Neural Network Control of Spacecraft Formation Using RISE Feedback," Advances in Neural Network Research and Applications, vol. 67, pp. 521- 528, 2010.

[23] J. Kennedy and R. C. Eberhart, "A new optimizer using particle swarm theory," in Proc. 1995 6th Int. Symp. Micro Machine Human Sci., Nagoya, Japan, pp. 39-43,

[24] M. I. Solihin, et al., "Comparison of LQR and PSO-based state feedback controller for tracking control of a flexible link manipulator," in 2nd IEEE International Conference on Information Management and Engineering (ICIME), 2010, pp. 354-358.

[25] A.R. Shirazi, M. M. S. Fakhrabadi, A. Ghanbari, "Optimal Design of a 6-DOF Parallel Manipulator Using Particle Swarm Optimization," Advanced Robotics, vol. 26, no. 13, 2012.

[26] E. Kim,”Output feedback tracking control of robot manipulators with model uncertainty via adaptive fuzzy logic," IEEE Trans. Fuzzy Systems vol. 12 no. 3, pp. 368378, 2004.

[27] M. Clerc and J. Kennedy, "The Particle SwarmExplosion, Stability, and Convergence in a Multidimensional Complex Space", IEEE Transactions on Eevolutionary Computation, vol. 6, no. 1, February, 2002.

\section{Authors' Profiles}

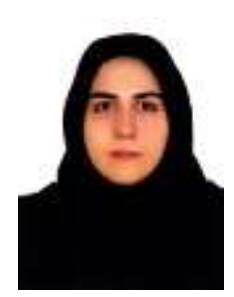

Marzieh. Yazdanzad was born in Sari, Iran in 1988. She received her B.Sc. degree in Power Electrical Engineering from the Faculty of Electrical and Computer Engineering, University of Mazandaran, Babol, Iran, in 2011. She is pursuing her M.Sc. degree in Control Engineering at the Babol University of Technology, Babol, Iran. 
Her research interests include Robust control, Intelligent control, Adaptive control and Artificial Intelligence applications to control systems.

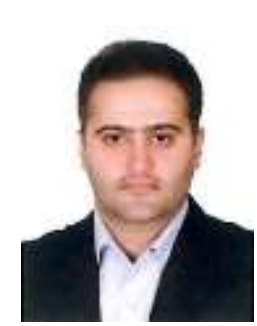

Alireza. Khosravi received the B.Sc. degree in Electrical Engineering from Mazandaran University, Babolsar, Iran. He received his M.Sc. and Ph.D. degrees in Control Engineering from Iran University of Science and Technology (IUST), Tehran, Iran, in 2004 and 2008, respectively. He is currently an assistant professor at Electrical Engineering Department, Babol University of Technology, Babol, Iran. His research interests include Robust Control, Optimal Control, modeling and system identification and intelligent systems.

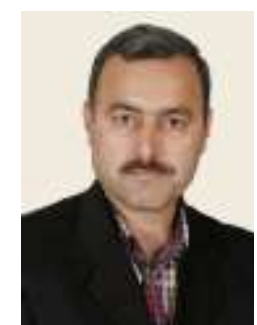

Abolfazl. Ranjbar Noei was born in Gorgan, Iran in 1964. He received B.Sc. degree from Isfahan University of Technology, Isfahan, Iran in 1988 and M.Sc. degree from Tarbiat Modaress University, Tehran, Iran in 1992, both in Control Engineering. He received his $\mathrm{Ph} . \mathrm{D}$. degree in Control Engineering from Surrey University, UK, in 2000. He is currently an associate professor at Electrical Engineering Department, Babol University of Technology, Babol, Iran. His research interests are Machine Control, Nonlinear and Chaos systems, Robust and Adaptive Control and Fractional Calculus. E-mail:.a.ranjbar@nit.ac.ir

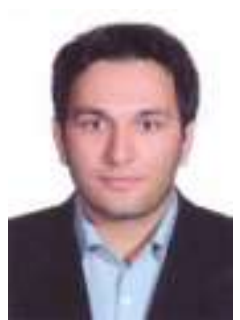

Pouria. Sarhadi received his B.Sc. degree in Electrical Engineering from Islamic Azad University, Ardabil Branch in 2006 and M.Sc. degree from Islamic Azad University south Tehran Branch in 2010. Currently, he is a Ph.D. student of Control Engineering at Babol University of Technology. His main research interests include adaptive control, robust control, system identification and systems engineering.

How to cite this paper: Marzieh Yazdanzad, Alireza Khosravi, Abolfazl Ranjbar N., Pouria Sarhadi,"Optimal Design of a RISE Feedback Controller for a 3-DOF Robot Manipulator Using Particle Swarm Optimization", International Journal of Information Technology and Computer Science(IJITCS), vol.6, no.8, pp.25-31, 2014. DOI: 10.5815/ijitcs.2014.08.04 